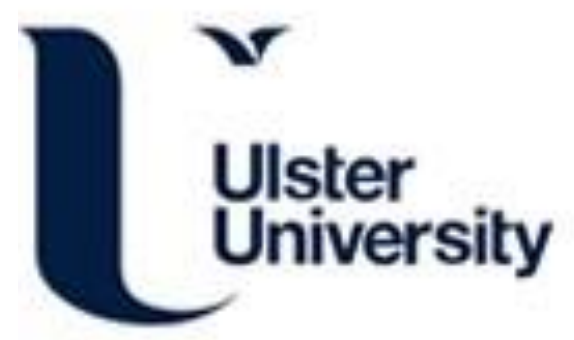

\title{
A qualitative study adopting a user-centered approach to design and validate a brain computer interface for cognitive rehabilitation for people with brain injury.
}

Martin, S., Armstrong, E., Thomson, E., Vargiu, E., Sola, M., Dauwalder, S., Miralles, F., \& Daly-Lynn, J. (2017). A qualitative study adopting a user-centered approach to design and validate a brain computer interface for cognitive rehabilitation for people with brain injury. Assistive Technology: The Official Journal of RESNA, 3, 1-9. https://doi.org/10.1080/10400435.2017.1317675

Link to publication record in Ulster University Research Portal

Published in:

Assistive Technology: The Official Journal of RESNA

Publication Status:

Published online: 14/07/2017

DOI:

10.1080/10400435.2017.1317675

\section{Document Version}

Author Accepted version

\section{General rights}

Copyright for the publications made accessible via Ulster University's Research Portal is retained by the author(s) and / or other copyright owners and it is a condition of accessing these publications that users recognise and abide by the legal requirements associated with these rights.

\section{Take down policy}

The Research Portal is Ulster University's institutional repository that provides access to Ulster's research outputs. Every effort has been made to ensure that content in the Research Portal does not infringe any person's rights, or applicable UK laws. If you discover content in the Research Portal that you believe breaches copyright or violates any law, please contact pure-support@ulster.ac.uk. 
Assistive Technology

The Official Journal of RESNA

\section{A qualitative study adopting a user-centered approach to design and validate a brain computer interface for cognitive rehabilitation for people with brain injury}

Suzanne Martin, Elaine Armstrong, Eileen Thomson, Eloisa Vargiu, Marc Solà, Stefan Dauwalder, Felip Miralles \& Jean Daly Lynn

To cite this article: Suzanne Martin, Elaine Armstrong, Eileen Thomson, Eloisa Vargiu, Marc Solà, Stefan Dauwalder, Felip Miralles \& Jean Daly Lynn (2017): A qualitative study adopting a usercentered approach to design and validate a brain computer interface for cognitive rehabilitation for people with brain injury, Assistive Technology, DOI: 10.1080/10400435.2017.1317675

To link to this article: http://dx.doi.org/10.1080/10400435.2017.1317675

Published online: 14 Jul 2017.

Submit your article to this journal $\sqsubset$

III Article views: 4

Q View related articles

View Crossmark data $₫$ 


\title{
A qualitative study adopting a user-centered approach to design and validate a brain computer interface for cognitive rehabilitation for people with brain injury
}

\author{
Suzanne Martin, $\mathrm{PhD}^{\mathrm{a}}$, Elaine Armstrong, $\mathrm{MSc}^{\mathrm{b}}$, Eileen Thomson, $\mathrm{MSc}^{\mathrm{b}}$, Eloisa Vargiu, $\mathrm{PhD}^{\mathrm{c}}$, Marc Solà, $\mathrm{MSc}^{\mathrm{c}}$, \\ Stefan Dauwalder, $\mathrm{MSc}^{c}$, Felip Miralles, $\mathrm{MSc}^{\mathrm{c}}$, and Jean Daly Lynn, MSc $\mathbb{1}^{\mathrm{b}}$
}

${ }^{a}$ Faculty of life and Health Sciences, Ulster University, Jordanstown, Northern Ireland, UK; ${ }^{b}$ Cedar Foundation, Belfast, Northern Ireland, UK; ceHealth Department, EURECAT, Barcelona, Spain

\begin{abstract}
Cognitive rehabilitation is established as a core intervention within rehabilitation programs following a traumatic brain injury (TBI). Digitally enabled assistive technologies offer opportunities for clinicians to increase remote access to rehabilitation supporting transition into home. Brain Computer Interface (BCI) systems can harness the residual abilities of individuals with limited function to gain control over computers through their brain waves.

This paper presents an online cognitive rehabilitation application developed with therapists, to work remotely with people who have $\mathrm{TBI}$, who will use $\mathrm{BCl}$ at home to engage in the therapy. A qualitative research study was completed with people who are community dwellers post brain injury (end users), and a cohort of therapists involved in cognitive rehabilitation. A user-centered approach over three phases in the development, design and feasibility testing of this cognitive rehabilitation application included two tasks (Find-a-Category and a Memory Card task). The therapist could remotely prescribe activity with different levels of difficulty. The service user had a home interface which would present the therapy activities. This novel work was achieved by an international consortium of academics, business partners and service users.
\end{abstract}

\section{ARTICLE HISTORY}

Accepted 30 March 2017

\section{KEYWORDS}

Cognitive impairment; computer access; information technology and telecommunications; neurorehabilitation and brain computer interfaces; service delivery

\section{Introduction}

It is estimated that 10 million people worldwide are affected every year by a traumatic brain injury (TBI; Hyder, Wunderlich, Puvanachandra, Gururaj, \& Kobusingye, 2007). TBI is a global public health problem affecting all populations and is predicted to become the third leading cause of mortality and disability globally by 2020 (The Lancet, 2012). A TBI is rapid in onset and may develop in a variety of circumstances; for example, trauma to the head, a vascular accident, cerebral anoxia, or infection (Tyerman \& Meehan, 2004). Following a BI or brain trauma, people may be left with a range of residual problems, which impact physical function, communication, cognition (thought processes), emotion, and behavior, requiring multidisciplinary rehabilitation to promote recovery and reintegration into society (Turner-Stokes, Disler, Nair, \& Wade, 2005). Scherer (2011) stated that cognitive disability is evident when standard and predictable thinking, remembering, learning, and behaving are altered. When a cognitive disability is present, a person is limited in their capacity to plan, sequence thoughts and actions, conceptualize ideas, and to interpret the meaning of social and emotional cues, and numbers and symbols (Braddock, Rizzolo, Thompson, \& Bell, 2004; Scherer, 2011). In this instance, cognitive rehabilitation is an important component of the therapeutic rehabilitation.

Cognitive rehabilitation is defined as a systematic, functionally orientated service of therapeutic activities based on assessment and understanding of the patient's brain-behavioral deficits directed toward many areas of cognition, including (but not necessarily limited to) attention, concentration, perception, memory, comprehension, communication, reasoning, problem solving, judgment, initiation, planning, self-monitoring, and awareness (Cicerone et al., 2000). Cognitive rehabilitation is core to the range of therapeutic interventions post-TBI-aiming to enhance independent everyday functioning, improve mobility, self-care, social interaction, and personal choice activities of recreation, school, work, and home management (Katz, Ashley, O'Shanick, \& Connors, 2006). Based on a comprehensive review of therapistdirected, evidence-based practice, Cicerone and colleagues (2000) considered it is clear that neuropsychological interventions for attention, memory, social communication skills, and executive function are empirically supported treatments for cognitive function (Cicerone et al., 2011). Evidence to support the use of computer-based rehabilitation programs has been growing for the last decade with examples extending to memory (Dou, Man, Ou, Zheng, \& Tam, 2006; Tam \& Man, 2004), working memory (Johansson \& Tornmalm, 2012; Lundqvist, Grundström, Samuelsson, \& Rönnberg, 2010), attention (Zickefoose, Hux, Brown, \& Wulf, 2013), and visual perception (Chen, Thomas, Glueckauf, \& Bracy, 1997; Hyun et al., 2009). Computer-based interventions are becoming increasingly popular because they often provide low cost, accessible, personalized therapies, with

CONTACT Suzanne Martin, PhD s.martin@ulster.ac.uk @ Room 1B114, School of Health Sciences, Faculty of Life and Health Science, Jordanstown Campus, Shore Road, Newtownabbey, County Antrim, BT37 OQB, Northern Ireland, UK.

Color versions of one or more of the figures in the article can be found online at www.tandfonline.com/UATY.

(c) 2017 RESNA 
immediate feedback, quantifiable outcomes, and indicated significant therapeutic benefits (Rego, Moreira, \& Reis, 2010). Interestingly, one research paper indicated the successful transfer of skills following the completion of a 20 -session problem-solving program on an interactive rehabilitation platform called PREVIRNEC remotely monitored by therapists (Soong \& Man, 2006). The initial findings indicated that $90.24 \%$ of participants with TBI reported some degree of cognitive improvement in a clinical trial of the cognitive tasks on the PREVIRNEC platform (Hernández et al., 2012).

Over the last 20 years, clinicians have been developing and evaluating technological interventions for individuals with TBI for everyday use and computer-based cognitive rehabilitation. Sometimes, however, due to the unique presentation of each person, access to systems remain evasive when the correct access method or switch to enable control cannot be found. Tai, Blain, and Chau (2008) described electroencephalography (EEG), electrocorticography, intracortical recordings, and electrodermal activity as emerging tools to harness residual capabilities in those living with no or very limited muscle movement. Brain computer interface (BCI) is one system for people with TBI-operated and controlled by EEG brain signals. Electrodes, which are non-invasive, are placed on the head to collect EEG signals generated by the user in response to a stimuli and send the information to the BCI so that it can be interpreted into a command (Kübler, Kotchoubey, Kaiser, Wolpaw, \& Birbaumer, 2001). Different paradigms have been developed offering a range of stimuli to control the BCI, with P300 the most commonly used paradigm (Guger, Krausz, Allison, \& Edlinger, 2012). BCI have proven to have wide-spanning functionality from the gaming industry to an assistive technology for home use (Miralles et al., 2015). Research illustrated that BCI systems can be controlled by individuals post-TBI (Schreuder et al., 2013). However, the impact a cognitive impairment has on EEG or the cognitive skills needed to operate a BCI are still unclear (Daly et al., 2015). Futher exploration is necessary, as people living with complex physical disability, and are in need of a technology solution like BCI, could also have a cognitive impairement.

The role of new technologies such as BCI within cognitive rehabilitation is expanding and expected to increase more rapidly in the future (Bamdad, Zarshenas, \& Auais, 2015). One of the visions set out for the future of BCI in the Horizon 2020 road map is that it could be used at home for rehabilitation (Brunner et al., 2015). BCI has been used as a cognitive rehabilitation training intervention in populations with attention deficit disorder (Lim et al., 2012) and older people (Lee et al., 2013). Both of these studies used the same BCI intervention to increase the users' attention skills through a three-dimensional video game as part of an attention training program. The findings indicated that both usability and acceptability of the program was high among users. No evidence was found of research undertaken to date incorperating a cognitive rehabilitation application onto a BCI for a population with TBI. The potential for cognitive rehabilitation applications through BCI post-TBI includes facilitating early intervention-particularly when a person is not able to move in a condition called locked-in syndrome (Smith \& Delargy, 2005).

Access to cognitive rehabilitation is important to maximize outcomes. Interestingly, Lee and his team (2013) are moving toward a larger trial of BCI cognitive training intervention to include people with early stage dementia. Exploring the different types of technologies and exploiting different types of human computer interaction opens up a range of possibilities and tools for clinicians to support their clients post-rehabilitation (How, Hwang, Green, \& Mihailidis, 2015). Therefore, access to cognitive rehabilitation through different modalities is important to maximize outcomes and enable a person to begin their rehabilitation journey through BCI at an earlier stage of recovery.

In this article, we focus specifically on developing a cognitive rehabilitation application on a BCI with therapist access through an online platform called a therapist station. This research adopted a user-centric design philosophy in the development of a rehabilitation tool that would focus on cognitive rehabilitation to complement available computer-based interventions. User-centered design is a process of engagement with target end users that adopts a range of methods to place those who may benefit most from the technology at the center of the design process in terms of development and evaluation (Kujala, 2003). The main stakeholders in this project are therapists and potential end users. Therapists include both occupational therapists (OTs) and speech and language therapists (SLTs), who work with people with TBI and prescribe treatment. End users are individuals living post-TBI who could potentially benefit from the developed system. The incremental system development in this study was based on user requirements and advice of the therapists prior to the system being used by the end users.

\section{Method}

In this qualitative research study, a user-centered approach based on three phases was adopted (Kujala, 2003) as illusrated in Figure 1. The first phase involved working with therapists to gather their requirements for the $\mathrm{BCI}$, with a cognitive rehabilitation application to be used at home post-TBI (phase 1). Secondly, SLTs and OTs engaged in an iterative development process with the design team to define the cognitive tasks (phase 2). Finally, the cognitive rehabilitation application was evaluated by a control group of people who did not have TBI and potential end users with TBI (phase 3). The Office for Research Ethics Northern Ireland (REC3) Ethics Committee provided ethical approval.

\section{Phase 1: Gathering user requirements}

The aim of the first phase was to understand what the therapists considered to be important in terms of the challenges of living with a TBI, the use of $\mathrm{BCI}$, and cognitive rehabilitation. During the initial gathering of user requirements, two focus groups were held. The first focus group brought together eight specialist neurological OTs with up to 20 years experience and all currently in clinical practice. The second focus group was much larger with a group of 58 newly qualified therapists. The overall aim was to harness the experienced therapists' clinical perspectives alongside the views of the younger therapists, who are considered to embrace technology more readily. Semi-structured questions were prepared in advance to guide the session. These included three broad themes. Theme 1: What is the impact of a neurological condition on a person in terms of their physical, cognitive, and mental health? Theme 2: Consider the impact of a 


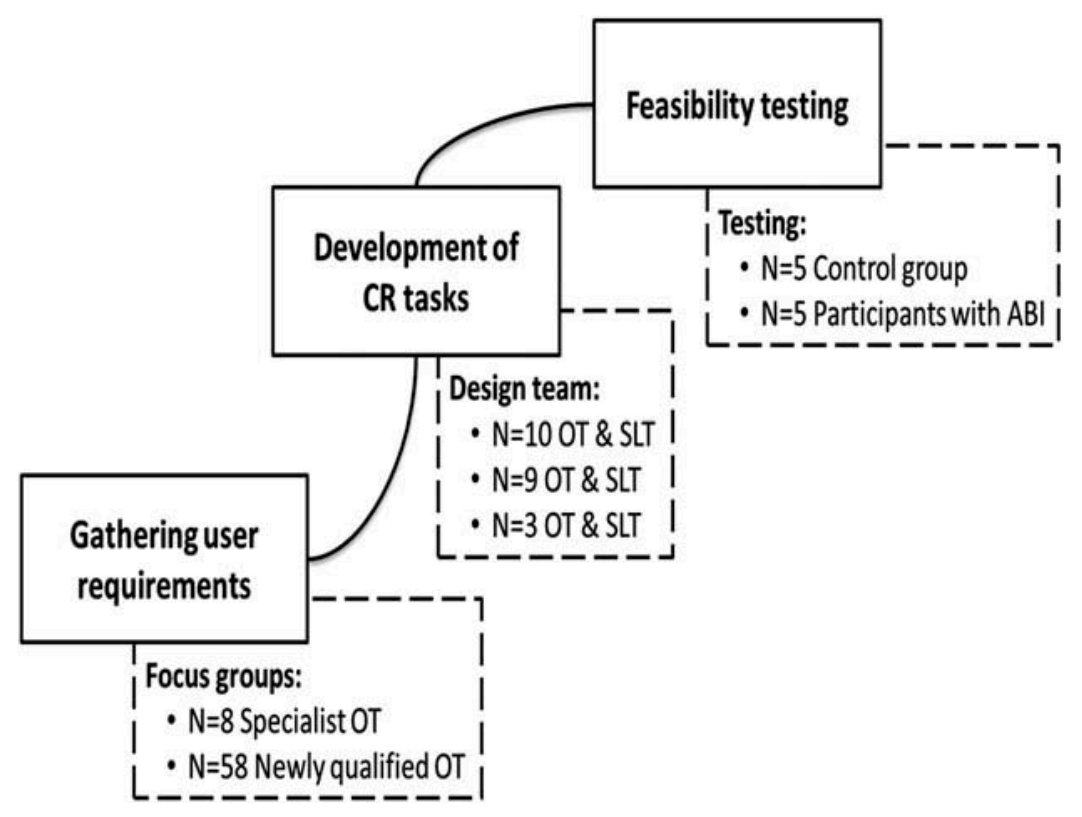

Figure 1. User-centered design approach.

Note. This iterative approach had three distinct phases to developing the cognitive rehabilitation tasks. First, it was necessary to find out what kind of tasks therapists would like; the tasks were then developed by the technical design team and refined by therapists over three sessions; and finally, the tasks were tested by a control and end-user group to identify the viability of cognitive rehabilitation on a $\mathrm{BCl}$ platform.

neurological condition on occupational performance, roles, and participation and engagement. Theme 3: BCI as an assistive technology for people with neurological problems living at home.

\section{Phase 2: Developing cognitive rehabilitation tasks}

The purpose of the second phase was to define and develop cognitive rehabilitation tasks for the BCI. Following on from the initial gathering of user requirements, a research collaboration was established with the specialist neurological OTs and SLTs. This group worked with the project team over a 1-year period to both develop and review the various stages of designing the cognitive rehabilitation application in advance of its integration into the BCI solution developed within BACKHOME. The therapists met with the researchers on three occasions in the rehabilitation center $(N=10 ; N=9 ; N=3)$. Each session lasted approximately 1 hour 30 minutes. At each session, a short powerpoint presentation was used to remind the therapists of the project aims and what would be covered in the session. Mock up slides of the graphical user interface and applications along with one BCI was also available. Technical support from the Spanish collaborator, EURECAT, was available remotely via Skype and TeamViewer applications if required. Following each session, the developers worked to integrate the feedback from the therapists into the cognitive rehabilitation application and move the application forward for the next session. The outcome from this iterative process was a cognitive rehabilitation application that would be used on a BCI.

\section{Phase 3: Feasibility testing}

This phase focused on the evaluation of efficacy and stability of the system. The two selected cognitive rehabilitation tasks that emerged from Phase 2 were evaluated by a control group and by end users with TBI. Ten people were recruited to evaluate the tasks: five participants (four female, $M=36.6$ years, \pm 9.3 ) in a control group, and five target end users (one female, $M=37$ years, \pm 8.7 ) living with TBI (Post-TBI $M=9.8$ years, \pm 3.7 ). Both groups completed the two integrated tasks at the easiest level of complexity on three separate occasions each. The target end users had a diagnosis of moderate to severe BI, were medically stable, and had no history of epilepsy in the last year or post-rehabilitation. Individuals' cognitive and physical impairment varied; however, each participant was able understand the study, give consent, and learn to interact with the BCI. No individual was living with locked-in syndrome at the time of testing; however, three participants lived experience of locked-in syndrome immediately after TBI for a period of time. All participants were ambulant, with different degrees of competency.

The evaluation took place in a rehabilitation center that was not controlled for environmental noise and the system was set up by non-computer experts. The tasks were scheduled by the researcher through the therapist station and were on the $\mathrm{BCI}$ interface for the participant to select prior to beginning the session. The therapist station was an online platform that could be accessed from any tablet or computer to schedule and receive the results of the cognitive rehabilitation tasks. The therapist station, BCI hardware, and software used in this study are described in detail in Miralles and colleagues (2015). The participant was asked to sit in front of a laptop, hosting the BCI based on Matlab software, that displayed the P300 control matrix. A second laptop, the user interface, was placed to the left of the BCI displaying the cognitive rehabilitation task (as shown in Figure 2). All selections were made through the P300 control matrix and the outcome could be viewed through the user interface. Each session began with training to create a unique classifer for the user. This meant the participant was required to select five predetermined letters from the P300 matrix. To make 


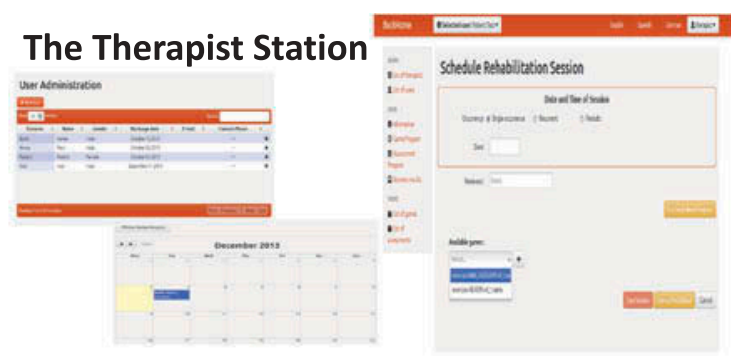

The BCl Prototype

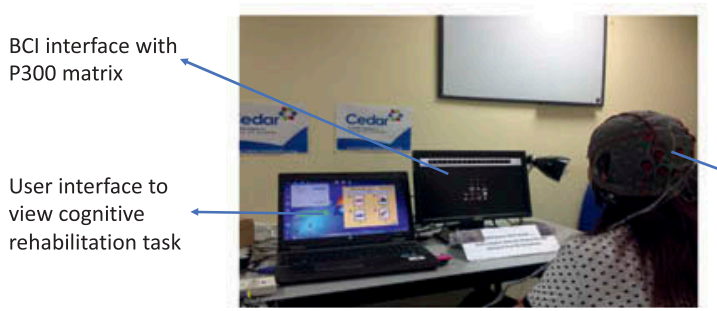

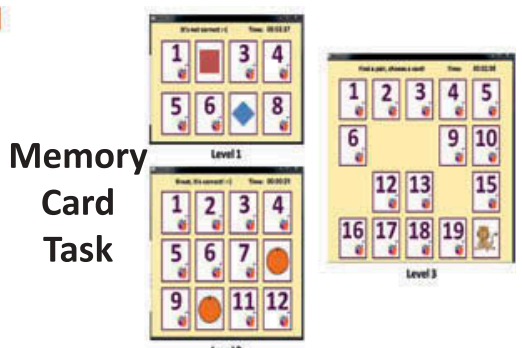

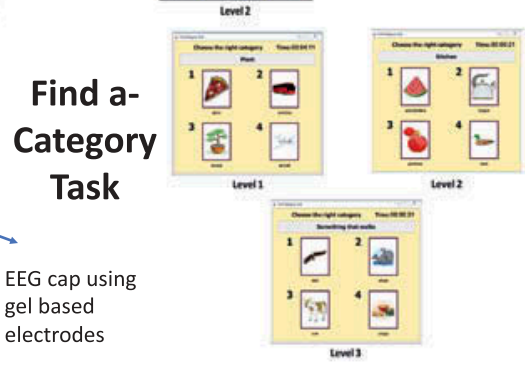

Figure 2. The therapist station, $\mathrm{BCl}$ prototype, and cognitive rehabilitation application.

Note. The therapist station can be accessed through any online device to schedule and receive the results from the cognitive rehabilitation task. The $\mathrm{BCl}$ prototype is controlled using the P300 matrix illustrated on the right screen, and the user interface displaying the cognitive rehabilitation application is on the left screen. The two cognitive rehabilitation tasks, find a category and memory task, both have three levels of difficulty.

a selection, users were required to focus on the predetermined letter and mentally count each time this symbol lit up when the rows and columns were flashing at random (see Guger et al., 2012 for more details on P300 BCI). The system can identify the desired symbol as a P300 brain wave, which will be elicited in response to the internal counting. The number of times the system detected the user's choice was recorded by the research team. The accuracy of the BCI was determined by calculating the percentage of intended selections made during the protocol to give a measure of technical accuracy, with a threshold set out at $<70 \%$ (Nijboer et al., 2008). Any additional technical issues observed during the session were documented by the research team. Participants were also given the opportunity to give feedback at the end of each testing session.

\section{Results}

Within this section, the results from each phase will be presented.

\section{Gathering user requirements}

Phase 1 was extremely useful in providing information on specific user requirements as perceived by the therapy staff from neurology, who work directly with people who have TBI. This information provided a backdrop for the researchers and developers for the design of the BCI, the therapist's station, and the initial approach to the cognitive rehabilitation tasks. In summary, these findings indicated that therapists wanted to remotely support clients. Interestingly, a number of practical requirements also emerged in terms of the BCI, such as the importance of a stylish EEG cap, the portability of the system, the capacity to focus on the screen for long periods, the necessary training required to use the system, and the reliability and dependability of the system. Additionally, in terms of the application, therapists queried how users would perceive this, who would be able to use it, how it would it be assessed, and would clients be able to understand it. Issues such as ethics, trust, and the potential limitations of real-life social interaction were also debated.

\section{Developing cognitive rehabilitation tasks}

Phase 2 focused on the specific development of the cognitive rehabilitation tasks. A framework for the rehabilitation of cognitive skills was created against which cognitive tasks were aligned during the collaborative stage of development. The framework for cognitive skills is shown in Figure 3. The domains emerging within the framework reflect various levels of cognitive complexity: perception, attention and concentration, memory, and executive functions. The sequential approach is important, as cognitive deficits at the lower levels of complexity impact on the person's competency to perform at the higher levels of complexity.

A therapist's station was developed to support the prescription of the tasks and to enable therapists to offer the continuation of care they indicated as important in Phase 1 (Vargiu et al., 2013). During each of the sessions, therapists were able to see the reality of having both a therapist station to prescribe cognitive rehabilitation and the end user interface that their service users would access via BCI. Therapists are able to interact with users in real time: monitor the use and outcomes of the cognitive rehabilitation tasks to attain therapeutic results. The station enables the therapist to plan, schedule, telemonitor, and personalize the prescription of cognitive rehabilitation tasks. It can also support therapists to monitor their clients' performance with the aim of motivating and supporting their progress.

During the iterative development process, therapists were asked to recommend tools they currently use within cognitive rehabilitation practice that would potentially be of value on the BCI system for clients. Cognitive rehabilitation tasks that represent real life living skills and language tasks were 


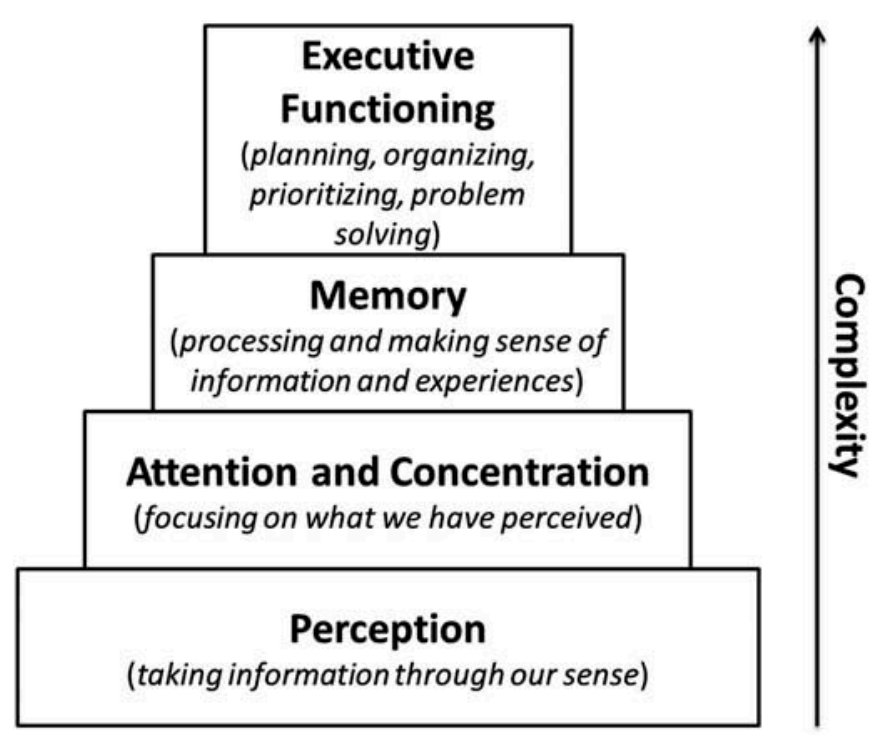

Figure 3. Cognitive skills pyramid.

Note. The cognitive skills pyramid provides a framework for the cognitive rehabilitation tasks to be developed within the project.

considered to be useful. Also freely available applications (apps) currently used by therapists were recommended. A number of proposed tasks were reviewed by developers and refined for a BCI application. The developers sent draft tasks to therapists that considered them in terms of usefulness of the task, difficulty levels, presentation and design, language, outcomes, sequencing, number of steps, and application to clinical practice. Recommendations to improve the tasks were often centered on visual processing and the need for the task design to be clear and simple without distracting images.

The presentation of results was recommended to include the score and speed of completion to be recorded, thus allowing the therapist to monitor and grade difficulty according to ability and performance. As a result of the collaborative process between the developers and therapists, the memory card task and find a category task were considered suitable for the BCI application.

The first implemented game on the BCI system was based on a memory card game and was aimed at enhancing memory skills on the framework in Figure 3. The task starts by showing all the cards turned face down on the user interface. In order to turn a card over to view the image, the user must make the selection on the BCI through the P300 matrix. The purpose of the task is to find each pair within the cards. The user chooses a card, which turns around to show an image, and then selects a second one to find a match for the first card. If the pair is not found, the cards are covered again. The game ends when all the pairs have been found. Three levels of difficulty were defined to support a range of end user abilities. The difficulty increased by increasing the number of available cards (i.e., the dimension of the P300 matrix [level $1=8$ cards; level $2=12$ cards; and level $3=20$ cards]) and the complexity of the adopted images (level 1 = shapes; level 2 = fruits; and level 3 =animals). The score is calculated by the numbers of correct moves divided by to the total number of moves, and the number of moves together with the elapsed time are illustrated on the screen. All the results are sent directly to the therapist through the therapist station.

The find a category game provides users with activities for improving semantic and reasoning skills essential in cognitive rehabilitation, language, and learning. Regarding the framework adopted in our work, this game aims to focus on attention-andconcentration skills. At each step, the game shows a category name and three objects. The goal is to identify which object belongs to the given category. Each game session is composed of 10 sets of category-objects. Different levels of difficulty have been defined depending on the ability of the user. At the first level, high-level categories are adopted (for example, "Plant"), whereas at the second one, more detailed ones are used (for example, "Kitchen"). As for the third level, an abstraction has been considered and the user has to make an inference to recognize the correct category. Examples of the adopted abstract categories are: "Cold Thing," "Hot Thing," "Made of Glass," "Made of Paper." The results for the task are shown to the user in the same way as the memory card task and are also sent to the therapist through the therapist station.

\section{Feasibility Testing}

The final stage included feasibility testing with the control group and those with TBI. Each participant completed the find a category task and the memory card task on three occasions all through the BCI. The results of this testing are presented in terms of the system functionality and the overall feedback for the developers of the cognitive rehabilitation application and the BCI design team. Within the two tasks, participants were not penalized for selecting the wrong answer. As long as the user made a selection and the BCI detected the choice, it was recorded as accurate; therefore, the number of selections made by the participants varied.

Overall, the control group achieved an average of $91.87 \%$ technical accuracy and end users achieved $78.13 \%$. This overall score is inclusive of the two steps necessary for the user to navigate to the prescribed tasks, and to complete the find a category task and the memory task. Two steps on the BCI matrix were requred by the user to navigate to the tasks sent from the therapist station. Once the cognitive rehabilitation icon is selected, first the find a category task is presented, and once completed, the memory task automatically begins. To achieve satisfactory control of the BCI, 70\% accuracy or above is required (Nijboer et al., 2008). To complete the find a category task, participants were asked five questions; therefore, if the user answers all the questions correctly and the system detects the intended choice each time, only five selections should have been needed to complete this task. The average technical accuracy score for the control group was $92.33 \%$, while the end user group achieved $75.2 \%$. Figure 4 outlines the individual accuracies for each participant over the three sessions. On average, the control group made six selections to complete the task compared to the end user group, who needed 7.7 selections to complete the task.

The accuracy scores for the memory tasks were slightly higher, with the control group reporting $92.93 \%$ and end users scoring $78.27 \%$. This task was more difficult to determine the amount of selections needed to complete the task, as 

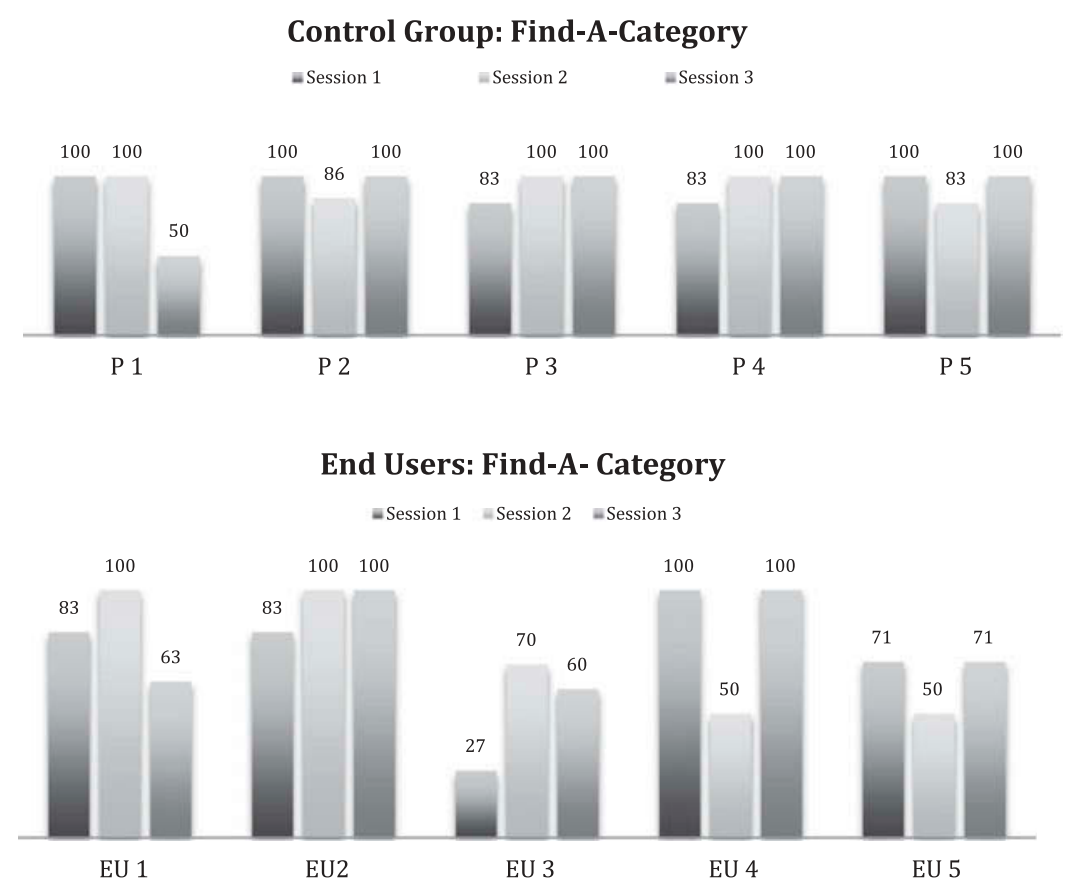

Figure 4. The individual accuracies for the find a category task per participant over the three sessions.

the user was required to remember and match the shapes at the underside of the card. The average selections necessary to compete the task for the control group overall was 14 . The average selections for the end users was 20.8, and this ranged quite significantly between 10 and 40 selections. Completing the cognitive rehabilitation tasks through the BCI added new technology to operate on one interface and the tasks on a separate interface. This added the dynamic of needing to split the client's attention between the two screens and the different types of focus, concentration, and memory needed to complete the task. It was found to be important to enable the end user to practice this task before using the BCI, as they found it confusing to remember what screen to look at, and at times frustrating. Equally, during the evaluation, the system had a number of stability problems. For example, if an error selection was made during the task, in some instances, the session was closed and it was necessary to restart the cognitive rehabilitation session from beginning. This meant that the scores accumulated every time the task was restarted.

The tasks received mixed reviews in particular from the end users due to the complexity of the tasks. The differences between the group's accuracy scores are not related to the number of correct or incorrect answers. However, they do relate to the participant's ability to complete cognitive rehabilitation on a BCI. The system often recorded a suppressed selection for the end user group, which reduced their accuracy score. A suppressed selection means that the system did not recognize their brain signal response to indicate their desired selection. This could be attributed to the end user spending longer attending to the user interface to process the answer before switching their attention to the P300 matrix to make their selection. The stimulus starts flashing automatically; thus, it is possible the end user would not have been attending to the P300 stimulus during the first number of flashing instances in time for the BCI to identify their selection. Figure 5 represents a summary of the results of this threephased user-centered approach.

\section{Discussion}

Cognitive recovery begins at different times and proceeds at different rates for people after TBI. A number of extensive reviews, recommendations, and guidelines on evidence-based cognitive rehabilitation are illustrated within the literature (Bragge et al., 2014). Within this research project, it has been our intention to work closely with rehabilitation therapists providing services to adults who have a TBI-proposing to deliver a novel system grounded in and relevant to clinical practice.

What emerged during our sessions is a strong desire on behalf of the therapists to find some means to support service users post-discharge beyond the annual review. Services are often provided in regional specialist centers, and post-discharge, both the therapist and the service user can feel abandoned. They consider this platform with a clinical GUI for them and therapeutic offerings to the service user as one way to possibly maintain contact. Research found that intensive, holistic, cognitive rehabilitation is an effective form of rehabilitation, particularly for persons with TBI, who have previously been unable to resume community functioning (Cicerone, Mott, Azulay, \& Friel, 2004). Perceived self-efficacy may have significant impact on functional outcomes after TBI rehabilitation. Measures of social participation and subjective well-being appear to represent distinct and separable rehabilitation outcomes after TBI. Cicerone and colleagues (2004) stated that satisfaction with cognitive functioning and community integration may reflect the perceived self-efficacy of a person with TBI regarding their functioning. It logically follows, therefore, that effort to develop the type of systems 


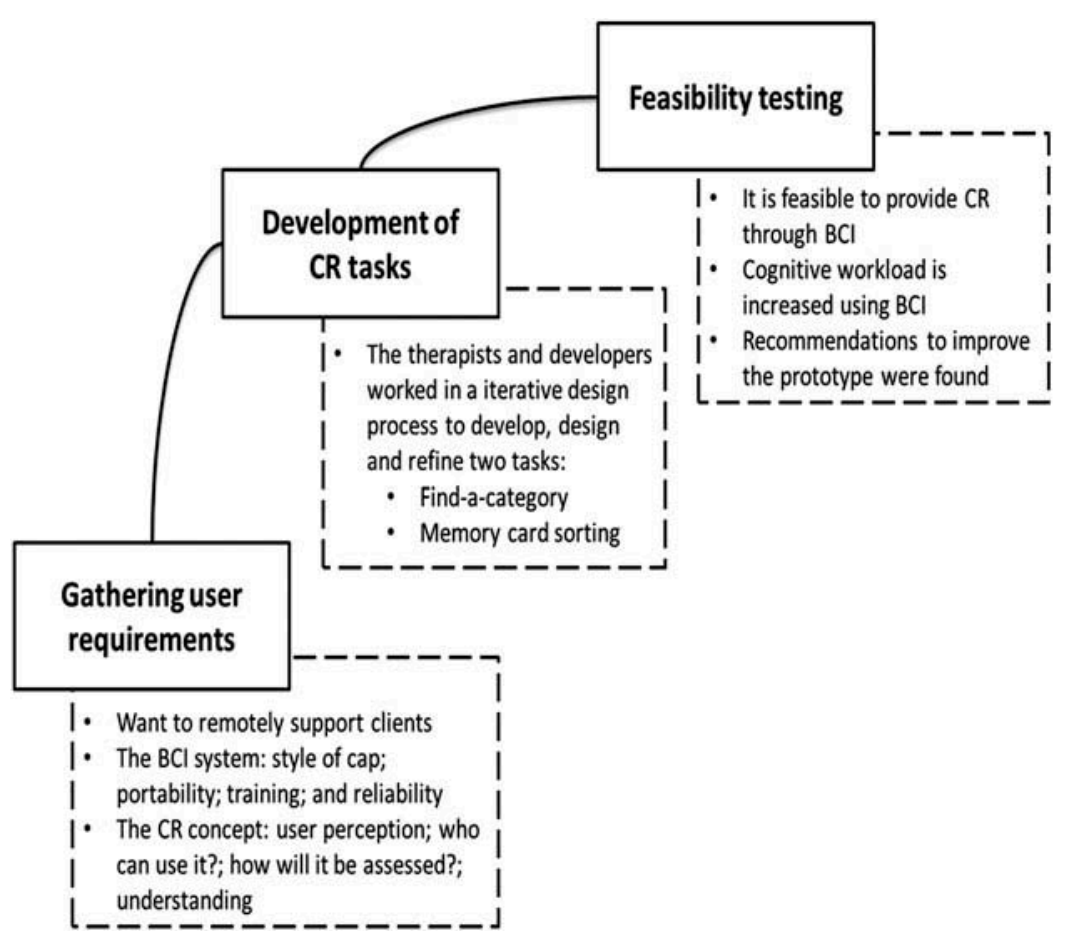

Figure 5. Summary of user-centered design approach.

Note. The outcome of the engagement process during each phase of the design and development of cognitive rehabilitation task for $\mathrm{BCl}$ is summerized.

described in this article may make a worthwhile contribution to the longer term outcomes for people with TBI if indeed they can support cognitive function and community integration.

There is no doubt that heterogeneity of TBI exists in terms of causes, pathophysiology, treatment, presentation, and outcome. Research highlighted that while this heterogeneity makes research in TBI particularly challenging, large international consortiums are needed, and also research between acute and post-acute care (Maas et al., 2013). Additionally, the need for both technical and therapeutic stakeholders in the development and design of new technology is essential to harness the new opportunities offered through these innovations (How et al., 2015). This research project brings together a consortium of European researchers from academia, industry, and non-government agencies to harness the essential perspectives (end users, health care providers, and engineers) to develop these complex interventions with those that will benefit from using them. A cognitive rehabilitation platform has been developed for integration into a BCI system or used as a stand-alone system.

The evaluation of the system with a control group and with people living with TBI suggested the potential for BCI as a platform for a cognitive rehabilitation application, and despite the technical issues that emerged, all participants were able to complete each of the cognitive rehabilitation tasks in excess of the recommended 70\% threshold for BCI accuracy (Nijboer et al., 2008). There were challenges using the $\mathrm{P} 300$ as the BCI paradigm; for example, establishing a unique user classification, which is prerequisite for usage. Also, some users found the speed of the P300 stimuli to be tiresome. However, the potential for BCI to be used as another modality for people living with TBI to engage with cognitive rehabilitation, such has been the case for populations with attention deficit disorder (Lim et al., 2012) and older people (Lee et al., 2013), offers huge promise to enable people at different stages on their path through rehabilitation. For people with significant physical and cognitive impairements post-TBI, these innovations offer hope of access to cognitive rehabilitation when other signals or modalities are not feasible.

Limitations of this work are inherent in the small sample size, and single site investigations in addition to the short time frame for exploitations. Furthermore, this research is aligned to prototype development and lacks any capacity to deliver evidence on effectiveness. Future research with empirical methods would be required to explore the BACKHOME $\mathrm{BCI}$ in terms of efficacy, effectiveness, impact, and value for money as a healthcare intervention. Additionally, further research would be useful to undertake an evaluation of the platform on which therapists will schedule and monitor their clients' therapy. Everyday technologies are pervasive and integrated into all our lives, and there are many opportunities to adopt and integrate technology into the lives of people with TBI. However, this will only be achieved if devices are useful, effective, reliable, available, and affordable.

\section{Conclusions}

Neurological conditions have unique presentations depending on the nature of the condition and site of injury to the brain. Personcentered rehabilitation will focus on the holistic needs of each person, which often includes the challenge of cognitive disability. The focus of this article was on the development, design, and feasibility testing of a cognitive rehabilitation program based on 
a BCI platform. The user-centered design approach enabled the European research consortium to incorporate the recommendations from end users in advance of entering a final phase of iterative change in the design of the system. The novelty of this system moves beyond the technical development to the remote provision of rehabilitation post-discharge by therapists on a system designed and tested by therapists and people with TBI.

\section{Conflict of Interest}

The authors claim to have no significant competing financial, professional, or personal interests that might have influenced the performance or presentation of the work described in this article.

\section{Acknowledgements}

The research leading to these results received funding from the European Community's Seventh Framework Programme FP7/2007-2013, BackHome project grant agreement $n^{\circ} 288566$.

\section{ORCID}

Jean Daly Lynn (D) http://orcid.org/0000-0001-9084-4677

\section{References}

Bamdad, M., Zarshenas, H., \& Auais, M. A. (2015). Application of BCI systems in neurorehabilitation: A scoping review. Disability and Rehabilitation: Assistive Technology, 10, 355-364. doi:10.3109/ 17483107.2014.961569

Braddock, D., Rizzolo, M. C., Thompson, M., \& Bell, R. (2004). Emerging technologies and cognitive disability. Journal of Special Education Technology, 19, 49-56.

Bragge, P., Pattuwage, L., Marshall, S., Pitt, V., Piccenna, L., StergiouKita, M., ... Bayley, M. (2014). Quality of guidelines for cognitive rehabilitation following traumatic brain injury. Journal Head Trauma Rehabilitation, 29, 277-289. doi:10.1097/HTR.0000000000000066

Brunner, C., Birbaumer, N., Blankertz, B., Guger, C., Kübler, A., Mattia, D., ... Opisso, E. (2015). BNCI Horizon 2020: Towards a roadmap for the BCI community. Brain-Computer Interfaces, 2, 1-10. doi:10.1080/ 2326263X.2015.1008956

Chen, S., Thomas, J., Glueckauf, R., \& Bracy, O. (1997). The effectiveness of computer-assisted cognitive rehabilitation for persons with traumatic brain injury. Brain Injury, 11, 197-210. doi:10.1080/026990597123647

Cicerone, K. D., Dahlberg, C., Kalmar, K., Langenbahn, D. M., Malec, J. F., Bergquist, T. F., ... Harrington, D. E. (2000). Evidence-based cognitive rehabilitation: Recommendations for clinical practice. Archives Physical Medica Rehabilitation, 81, 1596-1615. doi:10.1053/ apmr.2000.19240

Cicerone, K. D., Langenbahn, D. M., Braden, C., Malec, J. F., Kalmar, K., Fraas, M., ... Bergquist, T. (2011). Evidence-based cognitive rehabilitation: Updated review of the literature from 2003 through 2008 Archives Physical Medica Rehabilitation, 92, 519-530. doi:10.1016/j. apmr.2010.11.015

Cicerone, K. D., Mott, T., Azulay, J., \& Friel, J. C. (2004). Community integration and satisfaction with functioning after intensive cognitive rehabilitation for traumatic brain injury. Archives Physical Medica Rehabilitation, 85, 943-950. doi:10.1016/j.apmr.2003.07.019

Daly, J., Armstrong, E., Pinegger, A., Müller-Putz, G., \& Martin, S. (2015). In pursuit of an easy to use brain computer interface for domestic use in a population with brain injury. International Journal on Recent and Innovation Trends in Computing and Communication, 3(6), 4019-4029.

Dou, Z., Man, D., Ou, H., Zheng, J., \& Tam, S. (2006). Computerized errorless learning-based memory rehabilitation for Chinese patients with brain injury: A preliminary quasi-experimental clinical design study. Brain Injury, 20, 219-225. doi:10.1080/02699050500488215

Guger, C., Krausz, G., Allison, B. Z., \& Edlinger, G. (2012). Comparison of dry and gel based electrodes for P300 brain-computer interfaces. Frontiers Neuroscience, 6, 60. doi:10.3389/fnins.2012.00060

Hernández, R. C., Moreno, J. M., Molina, A. G., Celma, S. F., Sánchez, J. S., Carrión, R. S., ... Tafalla, C. A. (2012). 2D-tasks for cognitive rehabilitation. In 5th European Conference of the International Federation for Medical and Biological Engineering (pp. 838-841). Springer Berlin Heidelberg.

How, T., Hwang, A. S., Green, R. E., \& Mihailidis, A. (2015). Envisioning future cognitive telerehabilitation technologies: A co-design process with clinicians. Disability and Rehabilitation: Assistive Technology, 10, 1-18.

Hyder, A. A., Wunderlich, C. A., Puvanachandra, P., Gururaj, G., \& Kobusingye, O. C. (2007). The impact of traumatic brain injuries: A global perspective. NeuroRehabilitation, 22, 341-353.

Hyun, S. K., Kim, D. K., Kyung, M. S., Kwang, N. C., Jin, Y. Y., Sang, Y. S., \& Heon, J. P. (2009). A computerized visual perception rehabilitation programme with interactive computer interface using motion tracking technology-A randomized controlled, single-blinded, pilot clinical trial study. Clinical Rehabilitation, 23(5), 434-444. doi:10.1177/0269215508101732

Johansson, B., \& Tornmalm, M. (2012). Working memory training for patients with acquired brain injury: Effects in daily life. Scandinavian Journal of Occupational Therapy, 19, 176-183. doi:10.3109/ 11038128.2011 .603352

Katz, D., Ashley, M., O’Shanick, G., \& Connors, S. (2006). Cognitive rehabilitation: The evidence, funding and case for advocacy in brain injury. McLean, VA: Brain Injury Association of America.

Kübler, A., Kotchoubey, B., Kaiser, J., Wolpaw, J. R., \& Birbaumer, N. (2001). Brain-computer communication: Unlocking the locked in. Psychologist Bulletin, 127, 358. doi:10.1037/0033-2909.127.3.358

Kujala, S. (2003). User involvement: A review of the benefits and challenges. Behaviour \& Information Technology, 22, 1-16. doi:10.1080/ 01449290301782

Lee, T., Goh, S. J. A., Quek, S. Y., Phillips, R., Guan, C., Cheung, Y. B., ... Chin, Z. Y. (2013). A brain-computer interface based cognitive training system for healthy elderly: A randomized control pilot study for usability and preliminary efficacy. Plos One, 8, e79419. doi:10.1371/ journal.pone.0079419

Lim, C. G., Lee, T. S., Guan, C., Fung, D. S. S., Zhao, Y., Teng, S. S. W., ... Krishnan, K. R. R. (2012). A brain-computer interface based attention training program for treating attention deficit hyperactivity disorder. Plos One, 7, e46692. doi:10.1371/journal. pone. 0046692

Lundqvist, A., Grundström, K., Samuelsson, K., \& Rönnberg, J. (2010). Computerized training of working memory in a group of patients suffering from acquired brain injury. Brain Injury, 24, 1173-1183. doi:10.3109/02699052.2010.498007

Maas, A. I., Murray, G. D., Roozenbeek, B., Lingsma, H. F., Butcher, I., McHugh, G. S., ... Steyerberg, E. W.; International Mission on Prognosis Analysis of Clinical Trials in Traumatic Brain Injury (IMPACT) Study Group. (2013). Advancing care for traumatic brain injury: Findings from the IMPACT studies and perspectives on future research. The Lancet Neurology, 12, 1200-1210. doi:10.1016/S14744422(13)70234-5

Miralles, F., Vargiu, E., Rafael-Palou, X., Solà, M., Dauwalder, S., Guger, C., ... Martin, S. (2015). Brain computer interfaces on track to home: Results of the evaluation at disabled end-users's homes and lessons learnt. Frontiers in ICT, 2, 25. doi:10.3389/ fict.2015.00025

Nijboer, F., Sellers, E., Mellinger, J., Jordan, M., Matuz, T., Furdea, A., ... Vaughan, T. (2008). A P300-based brain-computer interface for people with amyotrophic lateral sclerosis. Clinical Neurophysiology, 119, 1909-1916. doi:10.1016/j.clinph.2008.03.034

Rego, P., Moreira, P. M., \& Reis, L. P. (2010). Serious games for rehabilitation: A survey and a classification towards a taxonomy. In 5th Iberian Conference on Information Systems and Technologies (CISTI) (pp. 1-6). New York, NY: IEEE. 
Scherer, M. J. (2012). Assistive technologies and other supports for people with brain impairment. New York, NY: Springer Publishing Company.

Schreuder, M., Riccio, A., Risetti, M., Dähne, S., Ramsay, A., Williamson, J., Mattia, D., \& Tangermann, M. (2013). User-centered design in brain-computer interfaces-a case study. Artificial Intelligence in Medicine, 59, 71-80.

Smith, E., \& Delargy, M. (2005). Locked-in syndrome. BMJ: British Medical Journal, 330(7488), 406.

Soong, Y. L. W., \& Man, W. K. D. (2006, Nov). A tele-cognitive rehabilitation platform for persons with brain injuries. Paper presented at TENCON 2006 IEEE Region 10 Conference, Hong Kong.

Tai, K., Blain, S., \& Chau, T. (2008). A review of emerging access technologies for individuals with severe motor impairments. Assistive Technology, 20(4), 204-221. doi:10.1080/10400435.2008.10131947

Tam, S., \& Man, W. (2004). Evaluating computer-assisted memory retraining programmes for people with post-head injury amnesia. Brain Injury, 18, 461-470. doi:10.1080/02699050310001646099
The Lancet. (2012). The changing landscape of traumatic brain injury research. Lancet Neurol, 11, 651. doi:10.1016/S1474-4422(12)70166-7 Turner-Stokes, L., Disler, P., Nair, A., \& Wade, D. (2005). Multidisciplinary rehabilitation for acquired brain injury in adults of working age. Cochrane Database Systems Reviews, 3, CD004170.

Tyerman, A., \& Meehan, M. (2004). Vocational assessment and rehabilitation after acquired brain injury: Inter-agency guidelines. Royal College of Physicians. Available from http://www.bsrm.org.uk/down loads/vocational-assessment-rehabilitation-abi.pdf

Vargiu, E., Fernández, J., Torrellas, S., Dauwalder, S., Sola, M., \& Miralles, F. (2013, September), . A sensor-based telemonitoring and home support system to improve quality of life through BNCI. Paper presented at 12th European AAATE Conference, Vila Moura, Portugal.

Zickefoose, S., Hux, K., Brown, J., \& Wulf, K. (2013). Let the games begin: A preliminary study using attention process training- 3 and Lumosity $^{\text {TM }}$ brain games to remediate attention deficits following traumatic brain injury. Brain Injury, 27, 707-716. doi:10.3109/ 02699052.2013.775484 